Pacific Journal of Mathematics

A MODIFICATION OF MORITA'S CHARACTERIZATION OF 


\section{A MODIFICATION OF MORITA'S CHARACTERIZATION OF DIMENSION}

\section{J. E. VAUGHAN}

Morita's characterization of dimension may be stated in the following form. Let $R$ be a metric space. A necessary and sufficient condition that $\operatorname{dim} R \leqq n$ is that there exists a $\sigma$-locally finite base $\mathscr{G}$ for the topology of $R$ such that $\operatorname{dim}$ $(\bar{G}-G) \leqq n-1$ for all $G$ in $\mathscr{G}$.

The main result of this paper is the following:

Theorem. Let $R$ be a metric space. A necessary and sufficient condition that $\operatorname{dim} R \leqq n$ is that there exists a $\sigma$ closure-preserving base $\mathscr{G}$ for the topology of $R$ such that $\operatorname{dim}(\bar{G}-G) \leqq n-1$ for all $G$ in $\mathscr{G}$.

Thus the "locally finite" condition in Morita's characterization can be replaced by the weaker "closure-preserving" condition. A further result is that the "closure-preserving" condition can be replaced by the still weaker condition of "linearly-closure-preserving" provided the "base" condition is strengthened to a "star-base" condition.

Finally, several examples are given which show that the "linearly-closure-preserving" condition is weaker than the "closure-preserving" condition in important ways. In particular, the following is proved.

Theorem. There exists a nonmetric, regular $T_{1}$-space which has a $\sigma$-linearly-closure-preserving star-base.

If the word "linearly" is deleted from the above theorem, the resulting statement is false since Bing has proved that a regular $T_{1}$-space with a $\sigma$-closure-preserving star-base is metrizable.

1. Introduction and results. Throughout this paper, $\operatorname{dim} R$ represents the usual covering dimension, and ind $R$ represents the small inductive dimension for a topological space $R$. See $[2 ; 3 ; 5]$.

Morita's well known characterization of dimension [5, Lemma 2.2, p. 351] states:

Let $R$ be a metric space. A necessary and sufficient condition that $\operatorname{dim} R \leqq n$ is that there exists a $\sigma$-locally finite base $\mathscr{G}$ for the topology of $R$ such that $\operatorname{dim}(\bar{G}-G) \leqq n-1$ for all $G$ in $\mathscr{G}$.

The main result of this paper is to modify Morita's result to: 
THEOREM 1. Let $R$ be a metric space. A necessary and sufficient condition that dim $R \leqq n$ is that there exists a $\sigma$-closure-preserving base $\mathscr{G}$ for the topology of $R$ such that $\operatorname{dim}(\bar{G}-G) \leqq n-1$ for all $G$ in $\mathscr{G}$.

Following the terminology of Michael [4], we say that a collection $\mathscr{G}$ of subsets of a topological space is closure-preserving provided that for every subcollection $\mathscr{B} \subset \mathscr{G}$ it is true that

$$
\cup\{\bar{B}: B \in \mathscr{B}\}=\overline{\cup\{B \in \mathscr{B}\}}
$$

A collection $\mathscr{G}$ of subsets is called $\sigma$-closure-preserving provided

$$
\mathscr{G}=\cup\left\{\mathscr{G}_{i}: i=1,2, \cdots\right\}
$$

with each $\mathscr{G}_{i}$ closure-preserving.

Instead of proving Theorem 1 directly, we shall prove a similar result, Theorem 2 , which has a weaker condition, but from which Theorem 1 can be proven easily. To facilitate the discussion of this and further results, we first make the following definitions.

DEFINITION. A collection $\mathscr{C}$ of subsets of a topological space is called linearly-closure-preserving provided that there exists a well ordering of $\mathscr{G}=\left\{G_{0}, G_{1}, \cdots, G_{\alpha}, \cdots: \alpha<\eta\right\}$ such that

$$
\cup\left\{\bar{G}_{\beta}: \beta<\alpha\right\}=\overline{\cup\left\{G_{\beta}: \beta<\alpha\right\}}
$$

for all $\alpha \leqq \eta$. A collection $\mathscr{G}$ of subsets of a topological space is called $\sigma$-linearly-closure-preserving provided $\mathscr{G}=\cup\left\{\mathscr{C}_{i}: i=1,2, \cdots\right\}$ with each $\mathscr{G}_{i}$ linearly-closure-preserving.

Definition. A collection $\mathscr{G}$ of open subsets of a topological space $R$ is called a $\sigma$-closure-preserving (respectively $\sigma$-linearly-closurepreserving) star-base for $R$ provided $\mathscr{G}=U\left\{\mathscr{G}_{i}: i=1,2, \cdots\right\}$ is a $\sigma$ closure-preserving (respectively $\sigma$-linearly-closure-preserving) collection such that for every point $x$ in $R$ and for every open set $D$ containing $x$ there exists a positive integer $k=k(x, D)$ such that

$$
\dot{\phi} \neq S\left(x, \mathscr{G}_{k}\right) \subset D,
$$

where $S\left(x, \mathscr{G}_{k}\right)=\cup\left\{G \in \mathscr{C}_{k}: x \in G\right\}$.

Theorem 2. Let $R$ bo a metric space. A necessary and sufficient condition that $\operatorname{dim} R \leqq n$ is that there exists a $\sigma$-linearly-closurepreserving star-base $\mathscr{G}$ for the topology of $R$ such that

$$
\operatorname{dim}(\bar{G}-G) \leqq n-1
$$


for all $G$ in $\mathscr{G}$.

The Nagata-Smirnov [7;9] characterization of metrizability for regular spaces (i.e., there exists a $\sigma$-locally finite base for the topology of the space) shows that Morita's result above can be modified to the following form:

Let $R$ be a regular $T_{1}$-space. A necessary and sufficient condition that $R$ be metrizable with $\operatorname{dim} R \leqq n$ is that there exists a $\sigma$-locally finite base $\mathscr{G}$ for the topology of $R$ such that

$$
\operatorname{dim}(\bar{G}-G) \leqq n-1
$$

for all $G$ in $\mathscr{G}$.

A similar modification of Theorern 1 is not possible. Bing has given [1, Example C, p. 180] a nonmetric, regular $T_{1}$-space which has a $\sigma$-closure-preserving base. Bing has proven, however, [1, Theorem 4 , p. 179] that a necessary and sufficient condition for a regular $T_{1}$ space to be metrizable is that there exists a $\sigma$-closure-preserving starbase for the topology of the space. Thus, as a direct result of Bing's Theorem and Theorem 1, we have:

TheOREM 3. Let $R$ be a regular $T_{1}$-space. A necessary and sufficient condition that $R$ be metrizable with $\operatorname{dim} R \leqq n$ is that there exists a $\sigma$-closure-preserving star-base $\mathscr{G}$ for the topology of $R$ such that $\operatorname{dim}(\bar{G}-G) \leqq n-1$ for all $G$ in $\mathscr{G}$.

Theorem 3 raises the question of whether one can replace " $\sigma$-closurepreserving" by " $\sigma$-linearly-closure-preserving" in Theorem 3 . This question is equivalent to the following one. Suppose a regular $T_{1}$ space $R$ has a $\sigma$-linearly-closure-preserving star-base; does this imply that $R$ is metrizable? The answer is in the negative as can be seen from the following example.

ExAMPle. A nonmetric, regular $T_{1}$-space which has a $\sigma$-linearlyclosure-preserving star-base. Let $C$ denote the usual "middle third" Cantor set in $[0,1]$, and let $Q$ denote the set of all rational points in $[0,1]$. The space $R$, which is to be the example, is the set of points of $C \cup Q$ with the following topology: $V$ is open in $R=C \cup Q$ if and only if $V=U \cup W$, where $U$ is open in the usual subspace topology of $R$, and $W$ is any set of irrational points in $R$. In this topology the irrational points of $R$ are discrete, and the topology induced on $Q$ is the usual subspace topology of $Q$. Now, $R$ is regular and $T_{1}$, but $R$ is not metrizable. 
To construct a $\sigma$-linearly-closure-preserving star-base for $\mathrm{R}$, we first enumerate the rational points of $R$ by $r_{1}, r_{2}, \cdots, r_{k}, \cdots$; and define

$$
\mathscr{G}_{i, j}=\left\{\left(r_{i}-1 / j, r_{i}+1 / j\right) \cap R\right\}
$$

for all $i, j \in N$ (where $N$ is the set of natural numbers). Since each $\mathscr{G}_{i, j}$ contains only one open set, it is trivially linearly-closure-preserving. We define one additional collection $\mathscr{G}_{0}=\left\{G_{0}, G_{1}, \cdots, G_{\alpha}, \cdots\right\}$ where $G_{0}=R-C$, and $\left\{G_{1}, G_{2}, \cdots, G_{\alpha}, \cdots\right\}$ is the set of irrational points in $R$ with any well ordering. Now $G_{0}$ is an open set in $R$ such that $G_{0} \cap C=\phi$ and $\bar{G}_{0} \cap C \supset Q$. From this it follows that the collection $\mathscr{G}_{0}$ is a linearly-closure-preserving collection of open sets. It is easily verified that the collections

$$
\mathscr{G}_{0} \cup\left(\cup\left\{\mathscr{G}_{i, j}: i, j \in N\right\}\right)
$$

can be ordered into a single countable sequence of collections, and as such form a $\sigma$-linearly-closure-preserving star-base for $R$.

Theorem 2 raises the question of whether one can replace "starbase" by "base" in Theorem 2. This question is easily answered in the negative as we now show. Roy [8] has defined a metric space $\Delta$ which has the property that $\operatorname{dim} \Delta=1$ and ind $\Delta=0$. Since ind $\Delta=0$, there exists a base $\mathscr{G}$ for $\Delta$ such that $\operatorname{dim}(\bar{G}-G)=-1$ for all $G$ in $\mathscr{G}$. If $\mathscr{G}$ is given any well ordering, and if the whole space $\Delta$ is added to the collection $\mathscr{G}$ as its first element, then $\mathscr{G}$ becomes a linearly-closure-preserving base for $\Delta$ such that $\operatorname{dim}(\bar{G}-\mathrm{G})$ $=-1$ for all $\mathrm{G}$ in $\mathscr{G}$. Since $\operatorname{dim} \Delta=1$, it is clear that "star-base" cannot be replaced by "base" in Theorem 2 .

2. Proof of Theorem 2. To prove the necessity of the condition, we note by Morita's result mentioned above that $\operatorname{dim} R \leqq \mathrm{n}$ implies that there exists a $\sigma$-locally finite base $\mathscr{G}=\cup\left\{\mathscr{G}_{i}: i \in N\right\}$ for $R$ such that $\operatorname{dim}(\bar{G}-G) \leqq n-1$ for all $G$ in $\mathscr{G}$. Since $R$.is a metric space, we may define

$$
\mathscr{G}_{i, k}=\left\{G \in \mathscr{G}_{i} \text { : diameter of } G<1 / k\right\}
$$

for all $i, k \in N$. Each $\mathscr{G}_{i, k}$ is locally finite (hence, linearly-closure-preserving), and $\operatorname{dim}(\bar{G}-G) \leqq n-1$ for all $G$ in $\mathscr{G}_{i, k}$ since $\mathscr{G}_{i, k} \subset \mathscr{G}_{i}$ for all $k$. By well ordering $\mathscr{G}^{\prime}=\cup\left\{\mathscr{G}_{i, k}: i, k \in N\right\}$ into a single countable sequence of collections, we have that $\mathscr{G}^{\prime}$ is a $\sigma$-linearly-closure-preserving star-base for $R$ such that $\operatorname{dim}(\bar{G}-G) \leqq n-1$ for all $G$ in $\mathscr{G}^{\prime}$.

The proof of the sufficiency will be broken up into several assertions. Each assertion will be assumed to have as hypothesis the condition of Theorem 2, i.e., $\mathscr{G}=\cup\left\{\mathscr{G}_{i}: i \in N\right\}$ is a $\sigma$-linearly-closurepreserving star-base for $R$ such that $\operatorname{dim}(\bar{G}-G) \leqq n-1$ for all $G$ 
in $\mathscr{G}$. The following notation and definitions will be used in the assertions.

For any subset $S$ of a topological space $R$, the boundary of $S$ is defined to be $\bar{S} \cap \overline{(R-S)}$, and is denoted by Bdry $(S)$.

Since each collection $\mathscr{G}_{i}$ is linearly-closure-preserving, we may write $\mathscr{G}_{i}=\left\{G_{i 0}, G_{i 1}, \cdots, G_{i \alpha}, \cdots: \alpha<\eta_{i}\right\}$ and define a collection of open sets by

$$
\left\{H_{i \alpha}=\left(G_{i \alpha}-\bigcup_{\beta<\alpha} \bar{G}_{i \beta}\right): \alpha<\eta_{i}\right\},
$$

and a collection of closed sets by

$$
\left\{F_{i \alpha}=\left(\bar{G}_{i \alpha}-\bigcup_{\beta \leqq \alpha} G_{i \beta}\right): \alpha<\eta_{i}\right\},
$$

and let

$$
\mathscr{H}_{i}=\left\{H_{i \alpha} \bigcap(R-F): \alpha<\eta_{i}\right\}
$$

for all $i \in N$, where $F$ is defined below.

2.1. Assertion. For all $i \in N, \bigcup\left\{F_{i \beta}: \beta<\alpha\right\}$ is a closed set in $R$ for every $\alpha \leqq \eta_{i}$.

Proof. Let $i$ be arbitrary, but fixed. Let $\alpha \leqq \eta_{i}$ and let $x$ be a limit point of $\cup\left\{F_{i \beta}: \beta<\alpha\right\}$. Then

$$
x \in \overline{\bigcup_{\beta<\alpha} F_{i \beta}}=\overline{\bigcup_{\beta<\alpha}\left(\bar{G}_{i \beta}-\bigcup_{\delta \leq \beta} G_{i \delta}\right)} \subset \overline{\bigcup_{\beta<\alpha} \bar{G}_{i \beta}} .
$$

Since the collection $\mathscr{G}_{i}$ is linearly-closure-preserving by hypothesis, $x \in \bigcup\left\{\bar{G}_{i \beta}: \beta<\alpha\right\}$. Let $\sigma<\alpha$ be the first index such that $x \in \bar{G}_{i \delta}$. It is easy to see that $x \notin G_{i \sigma}$, for $G_{i \sigma}$ is an open set which does not intersect $\bigcup\left\{F_{i \beta}: \sigma \leqq \beta<\alpha\right\}$. Hence, $x \in G_{i \sigma}$ would imply that $x$ is a limit point of $\bigcup\left\{F_{i \beta}: \beta<\sigma\right\}$. But this would imply that

$$
x \in \overline{\beta<\sigma} \overline{\mathrm{W}_{i \beta}} \subset \bigcup_{\beta<\sigma} \bar{G}_{i \beta},
$$

and this would mean that there exists $\delta<\sigma$ such that $x \in \bar{G}_{i \delta}$ which is impossible by the definition of $\sigma$. Hence, $x \notin G_{i \sigma}$. Thus, we have that

$$
x \in\left(\bar{G}_{i \sigma}-\bigcup_{\beta \subseteq \sigma} G_{i \beta}\right)=F_{i \sigma},
$$

and the assertion is proven.

The following notation will be used in the succeeding assertions. Let $F_{i}=\bigcup\left\{F_{i \beta}: \beta<\eta_{i}\right\}$, and let $F=\bigcup\left\{F_{i}: i \in N\right\}$. 


\subsection{Assertion. $\operatorname{Dim} F \leqq n-1$.}

Proof. By Assertion 2.1, $F_{i}$ is closed for all $i \in N$. Hence, it suffices by the usual sum theorem [5, Theorem 5.2, p. 355] to prove that $\operatorname{dim} F_{i} \leqq n-1$ for all $i$. Let $i$ be arbitrary, but fixed. Then by the subset theorem [5, Theorem 5.1, p. 355] we have that $\operatorname{dim} F_{i \alpha} \leqq n-1$ because

$$
F_{i \alpha} \subset\left(\bar{G}_{i \alpha}-G_{i \alpha}\right)
$$

and $\operatorname{dim}\left(\bar{G}_{i \alpha}-G_{i \alpha}\right) \leqq n-1$ by hypothesis. By Assertion 2.1

$$
\left\{F_{i \alpha}: \alpha<\eta_{i}\right\}
$$

is a linearly-closure-preserving collection such that $\operatorname{dim} F_{\imath \alpha} \leqq n-1$ for all $\alpha<\eta_{i}$. Hence, the collection $\left\{F_{i \alpha}: \alpha<\eta_{i}\right\}$ satisfys the hypothesis of a sum theorem of Nagami [6, Theorem 1, p. 82]. Thus,

$$
\operatorname{dim}\left(\bigcup\left\{F_{i \alpha}: \alpha<\eta_{i}\right\}\right) \leqq n-1
$$

and the assertion is proven.

To complete the proof of Theorem 2, we need only prove that $\operatorname{dim}(R-F) \leqq 0$ by [5, Theorem 5.4, p. 355]. To prove that

$$
\operatorname{dim}(R-F) \leqq 0
$$

it suffices by Morita's characterization of dimension to demonstrate a $\sigma$-discrete base for $R-F$ each member of which has an empty boundary in $R-F$.

2.3. Assertion. The collections $\mathscr{C}_{i}$ are discrete in the subspace $R-F$ for all $i \in N$.

Proof. Let $i$ be arbitrary, but fixed. We shall show that for every $x$ in $R-F$ there exists an open neighborhood of $x$ in $R-F$ which intersects at most one of the sets $H_{i \alpha} \cap(R-F)$. Let $x \in R-F$. If $x \notin \bigcup\left\{\bar{G}_{i \alpha}: \alpha<\eta_{i}\right\}$ then $R-\bigcup\left\{\bar{G}_{i \alpha}: \alpha<\eta_{i}\right\}$ is an open neighborhood of $x$ in $R$ which intersects none of the $H_{i \alpha}$, hence, none of the

$$
H_{i \alpha} \cap(R-F) \text {. }
$$

If, in the other case, $x \in \bigcup\left\{\bar{G}_{i \alpha}: \alpha<\eta_{i}\right\}$ let $\sigma<\eta_{i}$ denote the first index such that $x \in \bar{G}_{i \sigma}$. We may assume that $x \in G_{i \sigma}$, for otherwise,

$$
x \in\left(\bar{G}_{i \sigma}-\bigcup_{B \subseteq \sigma} G_{i \beta}\right)=F_{i \sigma} \subset F,
$$

which is impossible because $x \in R-F$. By the definition of $\sigma$ we see 
that

$$
x \in\left(G_{i \sigma}-\bigcup_{\beta<\sigma} \bar{G}_{i \beta}\right) \subset H_{\imath \sigma} .
$$

Clearly, $H_{i \sigma}$ is an open neighborhood of $x$ which does not intersect any $H_{i \alpha}$ for $\alpha \neq \sigma$. Hence, $H_{i \sigma} \cap(R-F)$ is the required neighborhood of $x$. This completes the proof of Assertion 2.3.

2.4. Assertion. The collection $\bigcup\left\{\mathscr{C}_{i}: i \in N\right\}$ is a base for the subspace $R-F$.

Proof. Let $x \in R-F$. Let $D$ be any open set in $R-F$ which contains $x$. Let $D^{\prime}$ be an open set in $R$ such that $D=D^{\prime} \cap(R-F)$. By hypothesis there exists an integer $k$ such that $\phi \neq S\left(x, \mathscr{G}_{k}\right) \subset D^{\prime}$. Let $\sigma<\eta_{k}$ be the first index such that $x \in G_{k \sigma}$, then $G_{k \sigma} \subset D^{\prime}$. Now, $x \notin \bigcup\left\{\bar{G}_{k \beta}: \beta<\sigma\right\}$ for otherwise, $x \in \bigcup\left\{\bar{G}_{k \beta}: \beta<\sigma\right\}$ would imply that there exists an index $\hat{o}<\sigma$ such that $x \in \bar{G}_{k \delta}$. Since $\delta<\sigma$, we would have that

$$
x \in\left(\bar{G}_{k \delta}-\bigcup_{\beta \leqq \delta} G_{k \beta}\right)=F_{k \delta} \subset F .
$$

This is impossible since $x \in R-F$. Thus

$$
x \in\left(G_{k \sigma}-\bigcup_{\beta<\sigma} \bar{G}_{k \beta}\right)=H_{k \sigma} .
$$

Hence, $x \in H_{k o} \cap(R-F)$, which is an open neighborhood of $x$ in $R-F$ and a subset of $D$. Assertion 2.4 is, therefore, proven.

2.5. Assertion. For each $i, \operatorname{Bdry}\left(H_{i \alpha}\right) \subset F_{i}$ for all $\alpha<\eta_{i}$.

Proof. Let $i$ be fixed, and let $\alpha<\eta_{i}$. Since $\mathscr{G}_{i}$ is a linearlyclosure-preserving collection of open sets,

$$
\operatorname{Bdry}\left(H_{i \alpha}\right)=\operatorname{Bdry}\left(G_{i \alpha}-\bigcup_{\beta<\alpha} \bar{G}_{i \beta}\right) \subset \bigcup\left\{\operatorname{Bdry}\left(G_{i \beta}\right): \beta \leqq \alpha\right\} .
$$

Let $x \in \operatorname{Bdry}\left(H_{i \alpha}\right)$. Since $\bigcup\left\{G_{i \beta}: \beta<\alpha\right\}$ is an open set which does not intersect $H_{i \alpha}$, we have that $x \notin \bigcup\left\{G_{i \beta}: \beta<\alpha\right\}$. Let $\delta \leqq \alpha$ be the first index such that $x \in \operatorname{Bdry}\left(G_{i \delta}\right)$. Then

$$
x \in\left(\bar{G}_{i \delta}-\bigcup_{\beta \leqq \delta} G_{i \beta}\right)=F_{i \delta} \subset F_{i} .
$$

2.6. Assertion. Bdry $\left(H_{i \alpha} \cap(R-F)\right)=\dot{\phi}$ in the subspace $R-F$ for all $i \in N$, and for all $\alpha<\eta_{i}$. 
Proof. This assertion follows from Assertion 2.5 and the fact that the boundary of $\left(H_{i \alpha} \cap(R-F)\right)$ with respect to the subspace $R-F$ is a subset of the boundary of $H_{i \alpha}$ with respect to the space $R$.

By Assertions 2.3, 2.4, and 2.6 we have shown that

$$
\mathscr{H}=\bigcup\left\{\mathscr{C}_{i}: i \in N\right\}
$$

is a $\sigma$-discrete base for $R-F$ such that $\operatorname{dim}(\bar{H}-H)=-1$ for all $H$ in $\mathscr{H}$. Hence, $\operatorname{dim}(R-F) \leqq 0$, and Theorem 3 is completely proven.

3. Proof of Theorem 1. The proof of the necessity of the condition is trivial.

To prove the sufficiency, let $\mathscr{G}$ be the $\sigma$-closure-preserving base for $R$ such that $\operatorname{dim}(\bar{G}-G) \leqq n-1$ for all $G$ in $\mathscr{G}$. By the same method as was used in the proof of the necessity of Theorem $2, \mathscr{G}$ may be "rearranged" into a $\sigma$-closure-preserving star-base. Thus the condition of Theorem 2 is satisfied. We may, therefore, conclude that $\operatorname{dim} R \leqq n$, and Theorem 1 is proven.

The author would like to thank Dr. J. H. Roberts and Dr. Keiô Nagami for their guidance in the preparation of this paper.

\section{REFERENCES}

1. R. H. Bing, Metrization of topological spaces, Canad. J. Math. 3 (1951), 175-186.

2. W. Hurewitz and H. Wallman, Dimension theory, Princeton, 1955.

3. M. Katetov, On the dimension of non-separable spaces I, Cehoslovack Mat. Z. 2(77) (1953), 333-368.

4. E. Michael, Another note on paracompact spaces, Proc. Amer. Math. Soc. 8 (1957), $822-828$.

5. Kiiti Morita, Normal families and dimension theory for metric spaces, Math. Annalen 128 (1954/1955), 350-362.

6. Keiô Nagami, Some theorems in dimension theory for non-separable spaces, J. Math. Soc. Japan 9 No. 1 (1957), 80-92.

7. J. Nagata, On a necessary and sufficient condition of metrizability, J. Inst. Polytech., Osaka City Univ., Sec. A. Math., Vol. 1, (1950), 93-100.

8. Prabir Roy, Failure of equivalence of dimension concepts for metric spaces, Bull. Amer. Math. Soc. 68 (1962), 609-613.

9. Yu. Smirnov, A necessary and sufficient condition for metrizability of a topological space, Doklady Akad. Nauk. SSSR. N. S. 77 (1951), 197-200.

Received December 15, 1964. This work was supported by the National Science Foundation, Grant GP-2065, and is taken from the author's doctorial dissertation, Duke University, 1965. 


\section{PACIFIC JOURNAL OF MATHEMATICS}

H. SAMELSON

Stanford University

Stanford, California

J. P. JaNs

University of Washington

Seattle, Washington 98105

\section{EDITORS}

J. DugundJI

University of Southern California Los Angeles, California 90007

RICHARD ARENS

University of California

Los Angeles, California 90024

\section{ASSOCIATE EDITORS}

E. F. BECKENBACH

B. H. NEUMANN

F. WOLF

K. YosidA

\section{SUPPORTING INSTITUTIONS}

UNIVERSITY OF BRITISH COLUMBIA

CALIFORNIA INSTITUTE OF TECHNOLOGY

UNIVERSITY OF CALIFORNIA

MONTANA STATE UNIVERSITY

UNIVERSITY OF NEVADA

NEW MEXICO STATE UNIVERSITY

OREGON STATE UNIVERSITY

UNIVERSITY OF OREGON

OSAKA UNIVERSITY

UNIVERSITY OF SOUTHERN CALIFORNIA
STANFORD UNIVERSITY

UNIVERSITY OF TOKYO

UNIVERSITY OF UTAH

WASHINGTON STATE UNIVERSITY

UNIVERSITY OF WASHINGTON

AMERICAN MATHEMATICAL SOCIETY CHEVRON RESEARCH CORPORATION TRW SYSTEMS

NAVAL ORDNANCE TEST STATION 


\section{Pacific Journal of Mathematics}

\section{Vol. 20, No. $1 \quad$ September, 1967}

Leonard Daniel Baumert, Extreme copositive quadratic forms. II ........ 1

Edward Lee Bethel, A note on continuous collections of disjoint

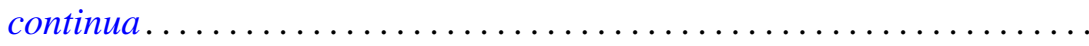

Delmar L. Boyer and Adolf G. Mader, A representation theorem for abelian groups with no elements of infinite p-height ...................

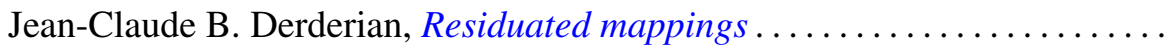

Burton I. Fein, Representations of direct products of finite groups ......... 45

John Brady Garnett, A topological characterization of Gleason parts.......

Herbert Meyer Kamowitz, On operators whose spectrum lies on a circle or

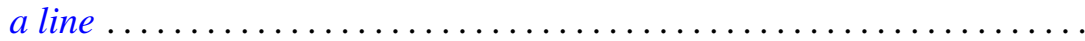

Ignacy I. Kotlarski, On characterizing the gamma and the normal distribution ........................................

Yu-Lee Lee, Topologies with the same class of homeomorphisms ..........

Moshe Mangad, Asymptotic expansions of Fourier transforms and discrete polyharmonic Green's functions ...........................

Jürg Thomas Marti, On integro-differential equations in Banach spaces ....

Walter Philipp, Some metrical theorems in number theory.............. 109

Maxwell Alexander Rosenlicht, Another proof of a theorem on rational

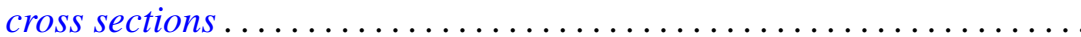

Kenneth Allen Ross and Karl Robert Stromberg, Jessen's theorem on Riemann sums for locally compact groups

Stephen Simons, A theorem on lattice ordered groups, results of Ptak, Namioka and Banach, and a front-ended proof of Lebesgue's theorem...

Morton Lincoln Slater, On the equation $\varphi(x)=\int_{x} x+1 K(\xi) f[\varphi(\xi)] d \xi \ldots$ 155 Arthur William John Stoddart, Existence of optimal controls .. 167 Burnett Roland Toskey, A system of canonical forms for rings on a direct sum of two infinite cyclic groups ....................

Jerry Eugene Vaughan, A modification of Morita's characterization of dimension 